\title{
LIMITS OF COVERING SPACES AND RESIDUAL PROPERTIES OF GROUPS
}

by JON MICHAEL CORSON

(Received 12 November, 1992)

1. Introduction. The purpose of this paper is to point out a flaw in H. B. Griffiths' covering space approach to residual properties of groups [3]. One is led to this paper from Lyndon and Schupp's book [4, pp. 114, 141] where it is cited for covering space methods and a proof that $F$-groups are residually finite. However the main result of [3] is false.

The problem is as follows. Given a group-theoretic property $\pi$, a group $G$ is said to be residually $\pi$ if for every element $g \neq 1$ of $G$ there exists a quotient group $\bar{G}$ which is a $\pi$-group such that the image of $g$ is non-trivial in $\bar{G}$. Equivalently, $G$ is residually $\pi$ if and only if the collection of normal subgroups $N$ of $G$ such that $G / N$ is a $\pi$-group has trivial intersection. For more details, see Magnus [5].

Consider the following situation. Suppose $G$ is a group and let a series

$$
G=G_{0} \supseteq G_{1} \supseteq G_{2} \supseteq G_{3} \supseteq \ldots
$$

of normal subgroups of $G$ be given. Then if each quotient $G / G_{n}$ is a $\pi$-group and the intersection of the terms of the series is trivial, it follows that $G$ is residually $\pi$.

To investigate the series (1) topologically, choose a locally 1-connected topological space $X$ such that $\pi_{1}(X) \cong G$ and form the sequence of covering spaces

$$
X=X_{0} \stackrel{\varphi_{1}}{\longleftarrow} X_{1} \stackrel{\varphi_{2}}{\longleftarrow} X_{2} \stackrel{\varphi_{3}}{\longleftarrow} \ldots
$$

corresponding to the series of subgroups (1). Denote the inverse limit of the system (2) of topological spaces and maps by $\lim X_{n}$. Let $J=\bigcap_{n=0}^{\infty} G_{n}$ and let $X_{J}$ be the covering space corresponding to the subgroup $J$.

In [3], Griffiths makes the claim that there is an injective homomorphism

$$
\breve{H}_{1}\left(X_{J}\right) \rightarrow \breve{H}_{1}\left(\underline{\lim } X_{n}\right)
$$

of the Čech homology groups with compact carriers and arbitrary coefficients, see Theorem 1 of [3]. However in Section 2 we given a counterexample to this.

As an alternative approach, we use singular homology. The corresponding assertion in singular homology is true and easy to prove, see Theorem 3.2. But the singular homology of the inverse $\operatorname{limit} \lim X_{n}$ is usually more difficult to compute. Čech homology has the computational advantage of being continuous in the sense that $\breve{H}_{1}\left(\lim X_{n}\right)=$ $\stackrel{\lim }{\longleftarrow} \check{H}_{1}\left(X_{n}\right)$, at least when the spaces involved are compact.

This technique is applied to study residual properties of groups as follows. Suppose the quotient $G / G_{n}$ is a $\pi$-group for each term in the series (1). Then if one can show the singular homology $H_{1}\left(\lim _{\longleftarrow} X_{n}\right)=0$, it follows that $H_{1}\left(X_{J}\right)=0$; that is, $J=\pi_{1}\left(X_{J}\right)$ is a

Glasgow Math. J. 36 (1994) 277-282. 
perfect group. And if $G$ is known to contain no non-trivial perfect subgroups, then $J=\bigcap_{n=0}^{\infty} G_{n}$ is trivial and hence $G$ is residually $\pi$. As a specific example, in Section 4 we give a covering space proof that free groups are residually finite $p$-groups for any prime $p$.

Notation. For convenience, all topological spaces are assumed to come with a preferred base point and if the space is a simplicial complex, the base point is at a vertex. The base point is then omitted from the notation of the fundamental group. Furthermore, all covering maps are assumed to be base point preserving.

If $G$ is a group, its abelianization is denoted $G^{\text {ab }}$. We use additive notation for $G^{\text {ab }}$, for example, $p . G^{\mathrm{ab}}$ is the subgroup of $p$ th powers in $G^{\mathrm{ab}}$. The torsion subgroup of $G^{\mathrm{ab}}$ is denoted $T(G)$. If $G$ is finitely generated, $T(G)$ has a free abelian complement in $G^{\text {ab }}$, i.e., $G^{\mathrm{ab}}=T(G) \oplus F(G)$ for some (non-unique) free abelian subgroup $F(G) \subseteq G^{\mathrm{ab}}$.

2. Counterexample. We make use of the following observation. Let $G$ be a finitely generated group. Then there exists a series as in (1) of subgroups with the properties:

(i) $G_{n} \ni G$,

(ii) $G_{n}$ has finite index in $G$,

(iii) The image of $G_{n+1}^{\mathrm{ab}} \rightarrow G_{n}^{\text {ab }}$ lies in $p . F\left(G_{n}\right)$, where $F\left(G_{n}\right)$ is some free abelian complement of $T\left(G_{n}\right)$ in $G_{n}^{\text {ab; }}$ thus $G_{n}^{\text {ab }}=T\left(G_{n}\right) \oplus$ $F\left(G_{n}\right)$.

The sequence is constructed inductively as follows. Let $G_{0}=G$. Having constructed $G_{0}, \ldots, G_{n}$, write $G_{n}^{\mathrm{ab}}=T\left(G_{n}\right) \oplus F\left(G_{n}\right)$ where $T\left(G_{n}\right)$ is the torsion subgroup and $F\left(G_{n}\right)$ is free abelian (note that (ii) implies $G_{n}$ is finitely generated). Then let $H \subseteq G_{n}$ be the subgroup corresponding to $p . F\left(G_{n}\right)$, so that $G_{n} / H \cong G_{n}^{\mathrm{ab}} / p . F\left(G_{n}\right)$. Then $H$ has finite index in $G_{n}$ and hence in $G$. So there exists a subgroup $G_{n+1} \subseteq H$ such that $G_{n+1}$ has finite index and is normal in $G$. Moreover the inclusion induced homomorphism $G_{n+1}^{\mathrm{ab}} \rightarrow G_{n}^{\mathrm{ab}}$ factors through $H^{\text {ab }}$ so its image lies in p.F( $\left.G_{n}\right)$. We see that $G_{n+1}$ satisfies (i)-(iii), as required.

Now, to make a counterexample to Griffiths' claim, start with a finitely presented solvable group $G$ that is not residually finite. For the existence of such groups, see Abels [1]. Construct a series of subgroups (1) satisfying (i)-(iii) as above. Choose a compact, connected simplicial complex $X$ such that $\pi_{1}(X) \cong G$. Then each space $X_{n}$, in the sequence of coverings (2) corresponding to the series of subgroups (1), is also compact as the $G_{n}$ have finite index in $G$. Furthermore $\breve{H}_{1}\left(X_{n}\right) \cong G_{n}^{\text {ab }}$ as $X_{n}$ is a simplicial complex, and the sequence of homology groups induced by (2) is naturally isomorphic to

$$
G^{\mathrm{ab}}=G_{0}^{\mathrm{ab}} \leftarrow G_{1}^{\mathrm{ab}} \leftarrow G_{2}^{\mathrm{ab}} \leftarrow \ldots
$$

where $G_{n+1}^{\mathrm{ab}} \rightarrow G_{n}^{\mathrm{ab}}$ is induced by the inclusion $G_{n+1} \subseteq G_{n}$.

We observe that conditions (i)-(iii) imply

(iv) $\lim _{-} G_{n}^{\mathrm{ab}}=0$.

To see this, let $\left(g_{n}\right)_{n=0}^{\infty}$ be an element of $\lim _{\leftarrow} G_{n}^{\mathrm{ab}}$. Fix an integer $n \geq 0$. Then for each 
integer $m \geq 0, g_{n}$ is in the image of $G_{n+m}^{\mathrm{ab}} \rightarrow G_{n}^{\mathrm{ab}}$. By (iii), this image lies in the subgroup $p^{m} . F\left(G_{n}\right)$. Hence each $g_{n} \in \bigcap_{m=0}^{\infty} p^{m} . F\left(G_{n}\right)=0$, so $\left(g_{n}\right)=0$ as required.

Now by the continuity of Čech homology (see Eilenberg-Steenrod [2]), $\breve{H}_{1}\left(\lim X_{n}\right) \cong$ $\lim _{\longleftarrow} \breve{H}_{1}\left(X_{n}\right) \cong \lim G_{n}^{\mathrm{ab}}=0$. Since $G$ is non-residually finite, $J=\cap G_{n} \neq 1$. But $G$ is solvable, so it contains no non-trivial perfect subgroups. Hence $\check{H}_{1}\left(X_{J}\right) \cong J^{\mathrm{ab}} \neq 0$ and there cannot be an injective homomorphism $\breve{H}_{1}\left(X_{J}\right) \rightarrow \breve{H}_{1}\left(\lim X_{n}\right)$. Therefore Griffiths' result is false in general.

3. The result in singular homology. Recall the setup of the introduction: a group $G$ and a series of subgroups (1) of $G$ are given; $X$ is a locally 1-connected topological space with $\pi_{1}(X)=G$ and we form the sequence (2) of covering spaces corresponding to the sequence of subgroups (1). (Assume throughout that all spaces are connected.)

We may assume that $X$ is a connected simplicial complex. Then the sequence (2) is an inverse system of simplicial complexes and simplicial maps. There are two ways to form the inverse limit of this system: either in the category of topological spaces or in the simplicial category. We now describe these and note that in general the results are different spaces.

The inverse limit in the topological category, which we denote by $\lim X_{n}$, is defined to be the subset of the cartesian product $\prod_{n=0}^{\infty} X_{n}$ consisting of the sequences $\left(x_{n}\right)_{n=0}^{\infty}$ such that $x_{n-1}=\varphi_{n}\left(x_{n}\right)$. There are continuous projections $q_{m}: \lim X_{n} \rightarrow X_{m}$ such that the diagrams:

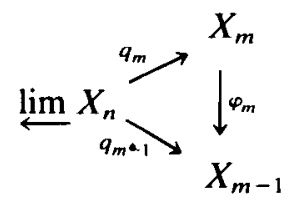

are commutative for all positive integers $m$. Indeed $\lim _{-} X_{n}$ and the projections are characterized by being universal among spaces and projections with this property.

The inverse limit of (2) in the simplicial category, denoted $\Delta$, is constructed as follows. The simplexes of $\Delta$ consist of sequences $\left(\sigma_{n}\right)_{n=0}^{\infty}$ where $\sigma_{n}^{\prime}$ is a simplex in $X_{n}$ and $\varphi_{n}\left(\sigma_{n}\right)=\sigma_{n-1}$. The face relation is given by: $\left(\sigma_{n}^{\prime}\right)$ is a face of $\left(\sigma_{n}\right)$ if $\sigma_{n}^{\prime}$ is a face of $\sigma_{n}$ in $X_{n}$ for each $n$. This collection of simplexes and the face relation form an (abstract) simplicial complex. Let $\Delta$ be the realization with the usual simplicial complex topology.

There is a natural simplicial (hence continuous) map $\Delta \rightarrow X_{n}$ for each $n$. These maps determine a continuous map $h: \Delta \rightarrow \lim _{\leftarrow} X_{n}$.

Remark 3.1. The map $h$ is easily seen to be bijective, though we don't use this fact. But $h$ is not necessarily a homeomorphism. For example, if each $X_{n}$ is a finite complex (i.e., compact) then it is well-known that $\lim _{\leftarrow} X_{n}$ is also compact. However if the number of simplexes in $X_{n}$ is unbounded as $n \rightarrow \infty$, then $\Delta$ is not compact (i.e., it has infinitely many simplexes). 
Recall that $J=\bigcap_{n=0}^{\infty} G_{n}$ and $X_{J} \rightarrow X$ is the covering space corresponding to the subgroup $J$. Since $J \subseteq G_{n}$, there is a unique base point preserving lift $X_{J} \rightarrow X_{n}$ for each $n$. These maps determine a map $\varphi: X_{J} \rightarrow \lim X_{n}$ into the inverse limit. Since the maps $X_{J} \rightarrow X_{n}$ are simplicial, they also determine a simplicial map $\theta: X_{J} \rightarrow \Delta$. Note that the composition

$$
X_{J} \stackrel{\theta}{\rightarrow} \Delta \stackrel{h}{\rightarrow} \lim _{\underline{-}} X_{n}
$$

is $\varphi$. We have the following.

TheOREM 3.2. The induced map $\varphi_{*}: H_{*}\left(X_{J}\right) \rightarrow H_{*}\left(\lim X_{n}\right)$ on singular homology with any coefficients is injective. Moreover, this map splits so $H_{*}\left(X_{J}\right)$ is a retract of $H_{*}\left(\lim X_{n}\right)$.

Proof. Let $\left|S\left(\lim _{\leftarrow} X_{n}\right)\right|$ be the geometric realization of the singular complex of $\lim _{\leftarrow} X_{n}$. Since $X_{J}$ is a simplicial complex, the map $\varphi: X_{J} \rightarrow \lim X_{n}$ factors through $\left|S\left(\lim X_{n}\right)\right|$ :

$$
X_{J} \stackrel{\varphi^{\prime}}{\rightarrow}\left|S\left(\lim _{\leftarrow} X_{n}\right)\right| \stackrel{\alpha}{\rightarrow} \lim _{\leftarrow} X_{n}
$$

where $\alpha:\left|S\left(\lim _{\longleftarrow} X_{n}\right)\right| \rightarrow \lim _{\longleftarrow} X_{n}$ is the canonical map. Now $\left|S\left(\lim X_{n}\right)\right|$ is a connected $C W$-complex and the map

$$
\pi_{1}\left(\left|S\left(\lim _{\leftarrow} X_{n}\right)\right|\right) \rightarrow \pi_{1}\left(\lim _{\leftarrow} X_{n}\right) \rightarrow \pi_{1}(X)
$$

factors through $\pi_{1}\left(X_{n}\right)=G_{n}$ for all $n$, so the image is in $\bigcap G_{n}=J$. Thus $\left|S\left(\lim _{\underline{m}} X_{n}\right)\right| \rightarrow X$ lifts to $X_{J}$. Then

$$
X_{J} \stackrel{\varphi^{\prime}}{\rightarrow}\left|S\left(\lim _{\llcorner} X_{n}\right)\right| \stackrel{\text { lift }}{\longrightarrow} X_{J}
$$

is a lift of the covering map $X_{J} \rightarrow X$. By uniqueness of lifts, the composition is the identity; whence $H_{*}\left(X_{J}\right) \rightarrow H_{*}\left(\left|S\left(\lim _{\leftarrow} X_{n}\right)\right|\right)$ is split injective. Since $\alpha_{*}: H_{*}\left(\left|S\left(\lim _{\leftarrow} X_{n}\right)\right|\right) \rightarrow H_{*}\left(\lim _{\leftarrow} X_{n}\right)$ is an isomorphism, the statement of the theorem follows.

Corollary 3.3. The induced map $\theta_{*}: H_{*}\left(X_{J}\right) \rightarrow H_{*}(\Delta)$ on singular (or simplicial) homology is injective.

Next consider the inverse limit $\lim _{\leftarrow} C\left(X_{n}\right)$ of the system of simplicial chain complexes induced by the sequence (2). The natural chain maps $C(\Delta) \rightarrow C\left(X_{n}\right)$ determine a chain $\operatorname{map} C(\Delta) \rightarrow \lim _{\leftarrow} C\left(X_{n}\right)$.

Lemma 3.4. The chain map $C(\Delta) \rightarrow \lim C\left(X_{n}\right)$ is injective.

Proof. Given a non-trivial chain $\sum n_{i} \sigma_{i}$ in $C(\Delta)$, notice that for sufficiently large $n$, the $\sigma_{i}$ are mapped by $C(\Delta) \rightarrow C\left(X_{n}\right)$ to distinct simplexes in $C\left(X_{n}\right)$. Whence the image of $\sum n_{i} \sigma_{i}$ is non-trivial in $\lim _{-} C\left(X_{n}\right)$.

The following consequence is used in the next section. 
Lemma 3.5. If $X$ is a 1-complex then there is an injective homomorphism

$$
H_{1}(\Delta) \rightarrow \lim _{\leftarrow} H_{1}\left(X_{n}\right)
$$

Proof. In this case, each $X_{n}$ and $\Delta$ are also 1-complexes so their 1st homology groups are equal to the simplicial 1-cycles: $H_{1}(\Delta)=Z_{1}(\Delta)$ and $H_{1}\left(X_{n}\right)=Z_{1}\left(X_{n}\right)$. Therefore the natural inclusion $C_{1}(\Delta) \rightarrow \lim C_{1}\left(X_{n}\right)$ of the previous lemma restricts to the desired monomorphism.

4. Application to free groups. As an application, we give a proof of a result due to Iwasawa:

Free groups are residually finite $p$-groups for any prime $p$.

Since finite $p$-groups are nilpotent, an immediate consequence is Magnus' theorem [6] that free groups are residually nilpotent, that is, the terms of the lower central series of a free group intersect trivially; also see [7, p. 314].

To prove Iwasawa's theorem, it clearly suffices to consider only finitely generated free groups. So let $G$ be a finitely generated free group and consider the series (1) constructed in Section 2. Since the abelianization of a free group is torsion free, the construction simplifies and it follows that for $n \geq 1, G_{n}=G^{p^{n}}[G, G]$ where $G^{p^{n}}$ is the subgroup jenerated by $p^{n}$ th powers of elements of $G$. That is, $G_{n}$ is the subgroup corresponding to $p^{\prime \prime} . G^{\mathrm{ab}}$. Observe that the series satisfies a stronger form of condition (ii), namely:

(ii') $G / G_{n} \cong G^{\mathrm{ab}} / p^{n} . G^{\mathrm{ab}}$ is a finite $p$-group.

The proof is completed by showing that $J=\bigcap_{n=0}^{\infty} G_{n}$ is trivial. For this, let $X$ be a finite graph (connected 1-complex) with $\pi_{1}(X) \cong G$ and form the sequence of covering spaces

$$
X=X_{0} \leftarrow X_{1} \leftarrow X_{2} \leftarrow X_{3} \leftarrow \ldots
$$

corresponding to the series in $G$ constructed above. Then the composite of the maps of

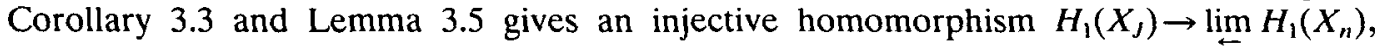
where $X_{J}$ is the covering space corresponding to the subgroup $J$. But $\lim H_{1}\left(X_{n}\right) \cong$ $\lim _{\leftarrow} G_{n}^{\mathrm{ab}}=0$ as noted in (iv) of Section 2 . Consequently $J^{\mathrm{ab}} \cong H_{1}\left(X_{J}\right)$ is trivial and hence $J$ is trivial because free groups contain no non-trivial perfect subgroups.

ACKNOWLedgement. I would like to thank the referee for simplifying and improving the original Theorem 3.2. Thanks also to the editor R. Steiner for correcting a critical error.

\section{REFERENCES}

1. H. Abels, An example of a finitely presented solvable group, Homological group theory, (ed. C. T. C. Wall), London Mathematical Society Lecture Note Series 36 (Cambridge University Press, 1979), pp. 205-211.

2. S. Eilenberg and N. Steenrod, Foundations of algebraic topology (Princeton University Press, Princeton, New Jersey, 1952). 
3. H. B. Griffiths, A covering-space approach to residual properties of groups, Michigan Math. J. 14 (1967), 335-348.

4. R. C. Lyndon and P. E. Schupp, Combinatorial group theory, Ergebnisse der Mathematik, Bd. 89 (Springer, New York, 1977).

5. W. Magnus, Residually finite groups, Bull. Amer. Math. Soc. 75 (1969), 305-316.

6. W. Magnus, Beziehungen zwischen Gruppen und Idealen in einem speziellen Ring, Math. Ann. 111 (1935), 259-280.

7. W. Magnus, A. Karrass, and D. Solitar, Combinatorial group theory, 2nd edition (Dover Publications, Inc., New York, 1976).

Department of Mathematics

UNIVERSITY OF ALABAMA

Tuscaloosa

Alabama 35487-0350

USA

E-mail: jcorson@ua1vm.ua.edu 